\title{
A Systematic Review and Meta-Analysis of Mindfulness-Based (Baduanjin) Exercise for Alleviating Musculoskeletal Pain and Improving Sleep Quality in People with Chronic Diseases
}

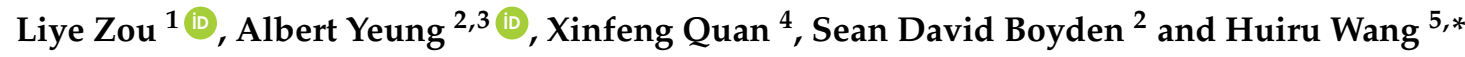 \\ 1 Department of Sports Science and Physical Education, The Chinese University of Hong Kong, \\ Hong Kong, China; liyezou123@gmail.com \\ 2 Depression Clinical and Research Program, Massachusetts General Hospital, Harvard University, \\ Boston, MA 02114, USA; AYEUNG@mgh.harvard.edu (A.Y.); SBOYDEN@partners.org (S.D.B.) \\ 3 The South Cove Community Health Center, Boston, MA 02111, USA \\ 4 Department of Material Science and Engineering, Sichuan University-Pittsburgh Institute, \\ Chengdu 610065, China; xinfeng.quan@gmail.com \\ 5 Department of Physical Education, Shanghai Jiaotong University, Shanghai 200240, China \\ * Correspondence: wanghrsjtu@163.com
}

Received: 25 December 2017; Accepted: 22 January 2018; Published: 25 January 2018

\begin{abstract}
Objective: we performed the first systematic review with meta-analyses of the existing studies that examined mindfulness-based Baduanjin exercise for its therapeutic effects for individuals with musculoskeletal pain or insomnia. Methods: Both English- (PubMed, Web of Science, Elsevier, and Google Scholar) and Chinese-language (CNKI and Wangfang) electronic databases were used to search relevant articles. We used a modified PEDro scale to evaluate risk of bias across studies selected. All eligible RCTS were considered for meta-analysis. The standardized mean difference was calculated for the pooled effects to determine the magnitude of the Baduanjin intervention effect. For the moderator analysis, we performed subgroup meta-analysis for categorical variables and meta-regression for continuous variables. Results: The aggregated result has shown a significant benefit in favour of Baduanjin at alleviating musculoskeletal pain (SMD $=-0.88,95 \% \mathrm{CI}-1.02$ to $-0.74, p<0.001, I^{2}=10.29 \%$ ) and improving overall sleep quality (SMD $=-0.48,95 \% \mathrm{CI}-0.95$ to $-0.01, p=004, I^{2}=84.42 \%$ ). Conclusions: Mindfulness-based Baduanjin exercise may be effective for alleviating musculoskeletal pain and improving overall sleep quality in people with chronic illness. Large, well-designed RCTs are needed to confirm these findings.
\end{abstract}

Keywords: mind-body exercise; Baduanjin Qigong; pain; sleep quality

\section{Introduction}

Musculoskeletal pain as a public health issue has been extensively investigated worldwide (e.g., in United States, Europe, Denmark, Singapore, Brazil, and Hong Kong) with respect to its prevalence [1-6]. Nine to forty percent of adults in the general population suffer from musculoskeletal pain (e.g., neck pain, shoulder pain, knee pain, low back pain, and widespread bodily pain), which was reported in these national-levels of epidemiological studies [1-6]. Of the adult population, individuals with chronic diseases (scapulohumeral periarthritis, ankylosing spondylitis, knee osteoarthritis, lumbar disc herniation, osteoporosis, and Type 2 diabetic mellitus) are more likely to have the complaints of these regional and widespread bodily pains [7-10]. Noteworthily, a high percentage of the symptomatic adults reported decreased work productivity, limited social participation, and poor sleep quality, ultimately leading to reduced quality of life [11]. According to the National Institute of Health [12], 
annual musculoskeletal pain-related expenses (e.g., ineffective treatment, over-the-counter medicine, medical doctor visits, the reduced number of working days, and decreased workability) had reached 65 billion US dollars; the economic burden of musculoskeletal pain is not only enormous for patients' families, but also challenges the national healthcare system.

Given the enormous financial burden and reduced quality of life in these symptomatic adults, musculoskeletal pain management as a medical and public health issue has drawn attention from the research community [13-16]. Although considerable effort has been made to investigate the effects of pharmacological treatment on alleviating musculoskeletal pain, its side effects after prolonged use of the drug therapies subsequently persist and may last for a lifetime [17-19]. Physical therapy and manual therapy as mainstream western non-pharmacological treatments are commonly recognized in medical settings, but they require a significant amount of time and money at an authorized rehabilitation center [20-23]. People living in developing or underdeveloped countries who have no health insurance could not afford these costly treatments. Thus, readily accessible and cost-effective intervention requires no limitations of time and space that is urgently demanded.

Chinese traditional Health-Qigong exercises (CTHQ) (e.g., Taichi, Baduanjin, Liu Zijue, and Wuqinxi), characterized by an integration of slow coordinating postures and musculoskeletal stretching movements, meditative mind, and breathing techniques have been extensively studied with respect to the health benefits [24-31]. Particularly, a recently published randomized controlled trial indicated that Tai-chi as one of the CTHQ was as effective as physical therapy for musculoskeletal pain management in 204 patients with knee osteoarthritis [32]. As compared to Tai-Chi, Baduanjin is an easy-to-learn health-Qigong form that can be independently practiced at home or in the workplace, because it only contains eight movements [33]. More importantly, Baduanjin involves musculoskeletal stretching throughout the entire body while applying breathing regulation and an empty state of mind; it seems to be more reasonable that Baduanjin may have musculoskeletal pain-alleviating effects [34]. Sleep disturbance was reported to be associated with elevated activation of the autonomic nervous system [35]. To reduce the activation levels in the autonomous nervous system, sleep quality could be improved through relaxed state of mind and breathing regulation during Baduanjin practice [36]. Although a high number of experimental studies were conducted in recent years to substantiate the proposal [37-42], a systematic review with a meta-analytical method on this topic has not been done so far. Thus, we will systematically and critically evaluate the emerging research literature related to the effects of Baduanjin on musculoskeletal pain and sleep quality in people with chronic diseases. Additionally, we want to determine if Baduanjin practice over more extended periods of time is more effective than a short term of Baduanjin. If the musculoskeletal pain could be alleviated after Baduanjin intervention, we assume that overall sleep quality in these symptomatic individuals would be improved as well.

\section{Methods}

\subsection{Search Strategy}

A comprehensive literature search was conducted by a review author (FXQ) through 3 September to 12 September 2017. The English-language electronic databases (PubMed, Web of Science, Elsevier, and Google Scholar) were used for the literature search. In addition, we also used two well-respected Chinese academic databases; they are Chinese National Knowledge Infrastructure (CNKI) and Wanfang. The purpose of this systematic review was to investigate the effects of Baduanjin on musculoskeletal pain and sleep quality. To obtain a maximum number of eligible articles, the search terms (Baduanjin or Eight-section Brocade) were separately integrated with the two interesting outcomes: "Baduanjin and pain", "Baduanjin and sleep quality", "eight-section Brocade and Pain", and "eight-section Brocade and sleep quality". 


\subsection{Inclusion Criteria and Study Selection}

To gain a comprehensive understanding of the effects of Baduanjin on musculoskeletal pain and sleep quality, all experimental studies (randomized controlled trial, RCT; non-randomized controlled study, NRCT; pretest-posttest study, PPS) were considered in this systematic review, but only RCT and NRCT were evaluated on the methodological quality, and their data were synthesized. Firstly, the experimental studies must be published in the peer-reviewed journals and full-text articles can be retrieved for data extraction. Secondly, each study must have no less than 10 adults who suffered at least one chronic disease (e.g., lower back pain, rheumatic disease, insomnia, Parkinson's disease, diabetic mellitus, or hypertension). Thirdly, at least one of the two outcomes (musculoskeletal pain and sleep quality) must be reported in these studies. Fourthly, Baduanjin should be the primary exercise intervention (e.g., Baduanjin vs. no training, Baduanjin + drug therapy vs. drug therpy, Baduanjin + acupuncture vs. acupuncture, or Baduanjin + educational program vs. educational program). Exclusion criteria included review studies, observational studies, and conference abstracts. A review author independently identified all potential articles against the inclusion criteria, which was verified by a second review author (review all papers as well). Discrepancies between the two review authors were discussed to reach their agreement.

\subsection{Assessment of Risk of Bias for Each Eligible Study}

Risk of bias for selected RCT and NRCT was interpedently assessed by a review author using the modified Physiotherapy Evidence Database (PEDro) Scale, followed by a verification of another review author (review all studies selected as well). The original 11-item PEDro scale involves eligibility criteria (does not contribute to total score), randomization, concealed allocation, similar baseline, blinding of all participants, blinding of all therapists, blinding of all assessors, more than $85 \%$ retention, intention-to-treat analysis (if missing data is present), between-group comparison, and point measures and measures of variability. We removed blinding of participants and Baduanjin instructors, because these two items are impractical in exercise intervention. Additionally, given the fact that prior sample size calculation and isolated Baduanjin intervention could affect the internal validity, we incorporated them into the modified assessment tool. If a criterion is clearly satisfied, one point was awarded and vice versa. Because Item-1 (eligibility criteria) does not contribute to total score, a sum score ranging from zero to ten can be awarded for each study, but summary quality scores were not used to categorize studies selected in this systematic, as suggested by Costa, Hilfiker, and Egger [43].

\subsection{Data Extraction and Synthesis}

The same review authors independently performed data extraction using a pre-created summary Table 1. The data extracted are study characteristics (the name of the leading authors, year of publication, and type of experimental design), study location and language of publication, initial sample size, dropout rate, study participants (disease condition with reporting of mean age or age range), intervention program (training duration and dosage, total training hours, weekly training hours, and total training sessions), test administration (outcome measured and blinding of assessor), and adverse event and follow-up assessment. For the two interesting outcomes, mean (M) and standard deviation (SD) at baseline and post-intervention from each eligible RCT and NRCT were extracted when the number of participants in experimental and control groups was obtainable. We tried to contact the corresponding author of one study because summary statistics were not reported, but received no response (thus, we excluded it).

Meta-analytical methods were used to synthesize the study findings of all eligible individual studies examining the effects of Baduanjin on musculoskeletal pain and/or sleep quality. Comprehensive Meta-Analysis Version 2.0 software (BioStat, Englewood, NJ, USA) was used to compute effect size (standardized mean difference (SMD) reflects the magnitude of the Baduanjin intervention effect) and the $95 \%$ confidence intervals (CI) while random effects model was set. 
With respect to musculoskeletal pain or sleep quality, the heterogeneity of effect sizes across all eligible studies was determined based on the $Q$-value. The expected $Q$-value is equivalent to or less than the degree of freedom (the number of studies minus one), indicating that the homogeneity of effect sizes across studies exists. The value of $I$-squared was used to determine the ratio of true heterogeneity to total observed variation. Specifically, $I$-squared ranging from 0 to $100 \%$ can be categorized into low heterogeneity ( $25 \%$ or lower), moderate heterogeneity ( 25 to $50 \%$ ), and high heterogeneity $(75 \%$ to $100 \%$ ). With respect to moderator analysis, we ran subgroup meta-analysis for categorical variables and meta-regression for continuous variables. In this systematic review, categorical variables include the final sample size of each eligible study ( $<60 \mathrm{vs.} \geq 60$ ), type of control groups (active (drug therapy, manual therapy, or other behavioral program) vs. non-active (no training, waitlist, original lifestyle, or usual care)), Baduanjin intervention length ( $<12$ weeks vs. $\geq 12$ weeks), weekly training hours $(<5 \mathrm{~h}$ per week vs. $\geq 5 \mathrm{~h}$ per week), and session length (less than or $45 \mathrm{~min}$ vs. $>45 \mathrm{~min}$ ). The number of total sessions and total hours of Baduanjin intervention is a continuous moderator. Additionally, publication bias was evaluated using the funnel plot and Egger's regression.

\section{Results}

\subsection{Literature Search}

Flow of literature search and selection process is shown in Figure 1. After both electronic and manual searches, 149 articles were initially identified. After exclusion of duplicates, irrelevant studies, review articles, and study protocols based on the titles and abstracts, 55 studies were left for further analysis. Of the remaining 55 studies, 27 full-text articles were excluded because of the following reasons: (1) healthy participants (with no diagnosed chronic conditions) $(n=12)$; (2) measurements of interest (pain and/or sleep quality) were not included $(n=4)$; (3) non-Baduanjin-based intervention (either incomplete Baduanjin used $(n=2)$ or other interventions were involved, e.g., medicine, manual therapy, or sports etc. $(n=6)) ;(4)$ incomplete data reported for analysis $(n=3)$. Our searches resulted in 28 eligible studies, including 25 RCTs, 2 NRCTs, and 1 PPS. When the similar baselines on the interesting outcomes were observed in the two NRCTs, we also considered them for meta-analysis in this study along with the 25 RCTs.

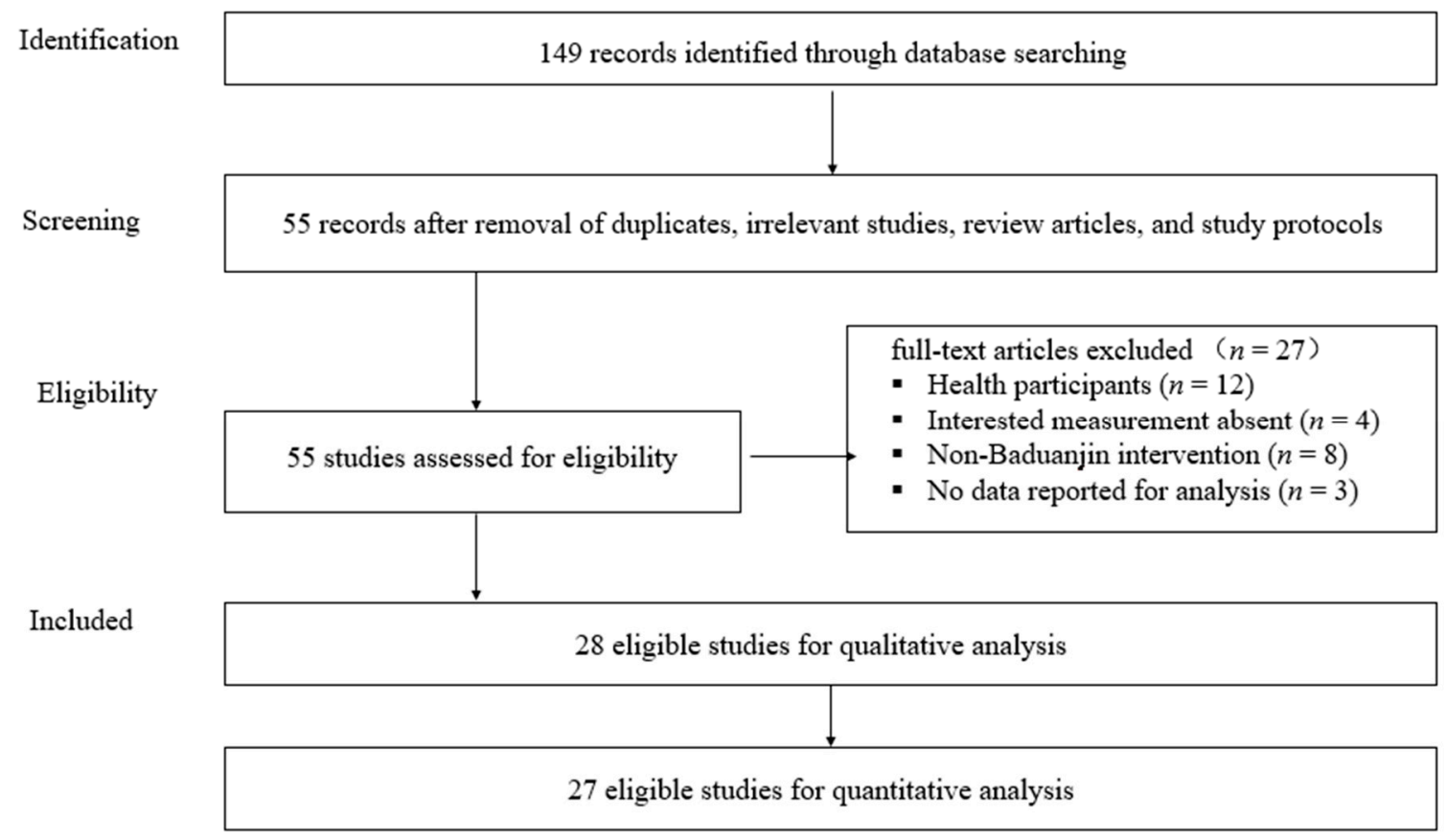

Figure 1. Flow of literature search and selection process. 


\subsection{Study Characteristics}

Table 1 shows the characteristics of the selected studies of 25 RCTs, 2 NRCTs, and 1 PPS, published between 2003 and 2017. Among the 28 studies, four were published in English, while the rest were in Chinese. All studies were carried out in mainland China except one, which was carried out in Hong Kong. Sample size of these studies ranged from 16 to 150 participants with 0 to $25 \%$ dropout rate. Collectively, this systematic review included a total of 1787 subjects with an age range of 15 to 80 .

The disease conditions involve insomnia, body pain (shoulder, neck, and/or back), periarthritis, ankylosing spondylosis, lumbar disc herniation, osteoporosis, type 2 diabetic mellitus, radiculopathy, Parkinson's disease, chronic fatigue syndrome-like illness, and hypertension accompanied with insomnia. Across all the $25 \mathrm{RCTs}$, the majority of the control groups received active interventions such as usual care, manual therapy, educational lessons, daily walking, drug therapy, acupuncture therapy (with or without cupping), and slinger exercise therapy, while one control group received no intervention and one control group waitlist. For the Baduanjin intervention groups, intervention times ranged from 2 weeks to 6 months, with 2 to 7 session per day in a week. A typical session lasted between $30 \mathrm{~min}$ to $90 \mathrm{~min}$. The number of total sessions in an individual study ranged from 9 to 336 , while the total session time ranged from 7 to $120 \mathrm{~h}$. One study did not report detailed information on number of sessions and total time but did report the time span of the intervention. Most studies included professional or trained (but not reporting whether they had earned certifications) Baduanjin instructors to teach participants or lead the intervention. Most frequently used instruments for the measurement of pain were the Visual Analog Scale (VAS), followed by the Short-form McGill Pain Questionnaire (SF-MPQ), subscale (pain) of the Western Ontario and McMaster Universities Osteoarthritis Index (WOMAC), subscale (pain) of the Self-Rating Anxiety Scale (SAS), and the Japanese Orthopedic Association Back Pain Evaluation Questionnaire (JOABPEQ). Two studies did not report instrument name for pain measurement. For the sleep quality measurement, only one study adopted the Parkinson's disease Sleep Scale, while the other 6 studies on sleep quality adopted the Pittsburgh Sleep Quality Index. Most studies reported no adverse events during the Baduanjin intervention, with 3 studies not mentioning adverse events. Only two studies reported follow-up assessment, 3 months and 6 months respectively, to track long term effect of Baduanjin on participants' pain and/or sleep quality improvement.

\subsection{Methodological Quality}

The inter-rater agreement was high $(95.4 \%)$ in selecting eligible studies. Methodological quality of all eligible studies was rated according to the modified PEDro scale, ranging from 4 to 8 points (Table 2). Of the 28 studies, none of them used the blinding of assessors and allocation concealment, which suggests fairly low quality. For the majority of the studies $(n=25,89.3 \%)$, risk of bias also came from the combination of Baduanjin intervention and other components (e.g., drug therapy, manual therapy, acupuncture, and education program). The other major source of risk of bias resulted from the absence of a priori sample size estimation $(n=27,96.4 \%)$. 
Table 1. Summary table relating to study.

\begin{tabular}{|c|c|c|c|c|c|c|c|c|c|c|c|c|}
\hline \multirow{2}{*}{$\begin{array}{c}\text { Author } \\
\text { [Reference] }\end{array}$} & \multirow{2}{*}{$\begin{array}{l}\text { Location, } \\
\text { Language }\end{array}$} & \multirow[b]{2}{*}{ ISZ (BJ/CG) } & \multirow[b]{2}{*}{ Drop-Out $\%$} & \multicolumn{2}{|c|}{ Study Participants } & \multicolumn{4}{|c|}{ Intervention Protocol } & \multicolumn{2}{|c|}{ Test Administration } & \multirow{2}{*}{$\begin{array}{l}\text { Adverse Event; } \\
\text { Follow-Up }\end{array}$} \\
\hline & & & & Health Status & $\begin{array}{l}\text { Mean Age or } \\
\text { Age Range }\end{array}$ & Training Duration and Dosage & $\begin{array}{c}\text { Total } \\
\text { Hours }\end{array}$ & $\begin{array}{c}\text { Hours } \\
\text { Weekly }\end{array}$ & $\begin{array}{c}\text { Total } \\
\text { Session }\end{array}$ & $\begin{array}{l}\text { Outcome } \\
\text { Measured }\end{array}$ & $\begin{array}{l}\text { Blinded } \\
\text { Assessor }\end{array}$ & \\
\hline $\begin{array}{l}\text { Du et al. [37], } \\
\text { RCT }\end{array}$ & $\begin{array}{l}\text { Qingdao, China } \\
\text { (Chinese) }\end{array}$ & $48(24 / 24)$ & $0 \%$ & $\begin{array}{l}\text { People with Discogenic low } \\
\text { back pain }\end{array}$ & $\begin{array}{l}\text { age range from } 38 \\
\text { to } 56\end{array}$ & $\begin{array}{c}\text { BJ: } 5 \times 40 \mathrm{~min} / \text { week for } 8 \text { weeks }+ \text { usual } \\
\text { care } \\
\text { CG: usual care }\end{array}$ & 27 & 3.33 & 40 & Pain (VAS) & NR & No/No \\
\hline $\begin{array}{l}\text { Wang et al. } \\
{[38], \text { RCT }}\end{array}$ & $\begin{array}{l}\text { Hangzhou, China } \\
\text { (Chinese) }\end{array}$ & $76(38 / 38)$ & $5.3 \%$ & $\begin{array}{l}\text { People with scapulohumeral } \\
\text { periarthritis }\end{array}$ & $\begin{array}{l}\text { Age range from } \\
40 \text { to } 66\end{array}$ & $\begin{array}{c}\text { BJ: } 5 \times 60 \mathrm{~min} / \text { week for } 4 \text { weeks }+ \text { usual } \\
\text { care } \\
\text { CG: usual care }\end{array}$ & 20 & 5 & 20 & Pain (VAS) & NR & No/No \\
\hline $\begin{array}{l}\text { Cai et al. } \\
\text { [39], RCT }\end{array}$ & $\begin{array}{l}\text { Changsha, China } \\
\text { (Chinese) }\end{array}$ & $60(30 / 30)$ & $0 \%$ & $\begin{array}{l}\text { patients with nerve-root type } \\
\text { cervical spondylosis }\end{array}$ & $\begin{array}{l}\text { A mean age } \\
\text { of } 50.4\end{array}$ & $\begin{array}{c}\text { BJ: } 7 \times 60 \mathrm{~min} / \text { week for } 6 \text { months }+ \\
\text { usual care } \\
\text { CG: usual care }\end{array}$ & 168 & 7 & 168 & Pain (VAS) & NR & No/No \\
\hline $\begin{array}{l}\text { Li et al. } \\
\text { [40], RCT }\end{array}$ & $\begin{array}{l}\text { Xi'an, China } \\
\text { (Chinese) }\end{array}$ & $\begin{array}{c}60 \\
(20 / 20 / 20)\end{array}$ & $0 \%$ & $\begin{array}{l}\text { Patients scapulohumeral } \\
\text { periarthritis }\end{array}$ & $\begin{array}{l}\text { A mean age } \\
\text { of } 50.43\end{array}$ & $\begin{array}{l}\text { BJ: } 5 \times 60 \mathrm{~min} / \text { week for } 6 \text { months } \\
\text { CG1: Twice per week (manual therapy) } \\
\text { CG2: Drug therapy: }\end{array}$ & 120 & 5 & 120 & Pain (NR) & NR & No/No \\
\hline $\begin{array}{l}\text { Xu et al. } \\
{[41], \text { RCT }}\end{array}$ & $\begin{array}{l}\text { Suzhou, China } \\
\text { (Chinese) }\end{array}$ & $16(8 / 8)$ & $0 \%$ & $\begin{array}{l}\text { Patients with lumbar disc } \\
\text { herniation }\end{array}$ & $\begin{array}{l}\text { A mean age } \\
\text { of } 48.1\end{array}$ & $\begin{array}{c}\text { BJ: } 7 \times 60 \mathrm{~min} / \text { week for } 1 \text { month }+ \text { drug } \\
\text { therapy } \\
\text { CG: drug therapy }\end{array}$ & 28 & 7 & 28 & Pain (VAS) & NR & No/No \\
\hline $\begin{array}{l}\text { Chen et al. } \\
\text { [42], RCT }\end{array}$ & $\begin{array}{l}\text { Chengdu, China } \\
\text { (Chinese) }\end{array}$ & $60(30 / 30)$ & $0 \%$ & $\begin{array}{l}\text { People with osteoporosis } \\
\text { suffered bodily pain }\end{array}$ & $\begin{array}{l}\text { Age range from } \\
55 \text { to } 71\end{array}$ & $\begin{array}{c}\text { BJ: } 7 \times 90 \mathrm{~min} / \text { week for } 8 \text { weeks }+ \text { usual } \\
\text { care } \\
\text { CG: usual care }\end{array}$ & 84 & 10.5 & 56 & Pain (VAS) & $\mathrm{NR}$ & No/No \\
\hline $\begin{array}{l}\text { Wei et al. } \\
{[44], \text { RCT }}\end{array}$ & $\begin{array}{l}\text { Liuzhou, China, } \\
\text { (Chinese) }\end{array}$ & $62(31 / 31)$ & $0 \%$ & $\begin{array}{l}\text { People with ankylosing } \\
\text { spondylitis }\end{array}$ & $\begin{array}{l}\text { age range from } 15 \\
\text { to } 60\end{array}$ & $\begin{array}{l}\text { BJ: } 7 \times 40 \text { min } / \text { week for } 3 \text { months }+ \text { drug } \\
\text { therapy (as same below) } \\
\text { CG: drug therapy }\end{array}$ & 56 & 4.67 & 84 & Pain (VAS) & NR & No/No \\
\hline $\begin{array}{l}\text { Wan et al. } \\
\text { [45], RCT }\end{array}$ & $\begin{array}{l}\text { Shanghai, China } \\
\text { (Chinese) }\end{array}$ & $60(30 / 30)$ & $0 \%$ & $\begin{array}{l}\text { People with myofascial pain } \\
\text { syndrome on shoulder and } \\
\text { neck }\end{array}$ & $\begin{array}{l}\text { A mean age } \\
\text { of } 41.92\end{array}$ & $\begin{array}{c}\text { BJ: } 7 \times 60 \mathrm{~min} / \text { week for } 35 \text { days }+ \\
\text { manual therapy (as same below) } \\
\text { CG: } 20 \text { min manual therapy for } 6 \text { sessions }\end{array}$ & 35 & 7 & 35 & $\begin{array}{l}\text { Pain (VAS } \\
\text { and SF-MPQ) }\end{array}$ & NR & No/No \\
\hline $\begin{array}{l}\text { Wang et al. } \\
{[46], \text { RCT }}\end{array}$ & $\begin{array}{l}\text { Hangzhou, } \\
\text { China, } \\
\text { (Chinese) }\end{array}$ & $67(34 / 33)$ & $3 \%$ & $\begin{array}{l}\text { People with scapulohumeral } \\
\text { periarthritis }\end{array}$ & $\begin{array}{l}\text { Age range from } \\
40 \text { to } 66\end{array}$ & $\begin{array}{c}\text { BJ: } 5 \times 60 \mathrm{~min} / \text { week for } 3 \text { months }+ \\
\text { usual care } \\
\text { CG: usual care }\end{array}$ & 60 & 5 & 60 & Pain (VAS) & NR & NR/No \\
\hline $\begin{array}{l}\text { Fu et al. } \\
{[47], \text { RCT }}\end{array}$ & $\begin{array}{l}\text { Langzhou, China } \\
\text { (Chinese) }\end{array}$ & $70(35 / 35)$ & $0 \%$ & $\begin{array}{l}\text { People with ankylosing } \\
\text { spondylitis }\end{array}$ & $\begin{array}{l}\text { Age range from } \\
17 \text { to } 42\end{array}$ & $\begin{array}{l}\text { BJ: } 7 \times 60 \mathrm{~min} / \text { week for } 6 \text { months }+ \text { drug } \\
\text { therapy (as same below) } \\
\text { CG: drug therapy }\end{array}$ & 168 & 7 & 168 & Pain (VAS) & NR & No/No \\
\hline $\begin{array}{l}\text { Li et al. } \\
\text { [48], RCT }\end{array}$ & $\begin{array}{l}\text { Jinan, China } \\
\text { (Chinese) }\end{array}$ & $60(30 / 30)$ & $0 \%$ & $\begin{array}{l}\text { People with chronic low back } \\
\text { pain }\end{array}$ & $\begin{array}{l}\text { Age range from } \\
35 \text { to } 60\end{array}$ & $\begin{array}{l}\text { BJ: } 5 \times 60 \mathrm{~min} / \text { week for } 8 \text { weeks } \\
\text { CG: Sling exerciser therapy }\end{array}$ & 40 & 5 & 40 & Pain (VAS) & $\mathrm{NR}$ & No/6-month \\
\hline
\end{tabular}


Table 1. Cont.

\begin{tabular}{|c|c|c|c|c|c|c|c|c|c|c|c|c|}
\hline \multirow{2}{*}{$\begin{array}{c}\text { Author } \\
\text { [Reference] }\end{array}$} & \multirow{2}{*}{$\begin{array}{l}\text { Location, } \\
\text { Language }\end{array}$} & \multirow[b]{2}{*}{ ISZ (BJ/CG) } & \multirow[b]{2}{*}{ Drop-Out $\%$} & \multicolumn{2}{|c|}{ Study Participants } & \multicolumn{4}{|c|}{ Intervention Protocol } & \multicolumn{2}{|c|}{ Test Administration } & \multirow{2}{*}{$\begin{array}{l}\text { Adverse Event; } \\
\text { Follow-Up }\end{array}$} \\
\hline & & & & Health Status & $\begin{array}{l}\text { Mean Age or } \\
\text { Age Range }\end{array}$ & Training Duration and Dosage & $\begin{array}{c}\text { Total } \\
\text { Hours }\end{array}$ & $\begin{array}{c}\text { Hours } \\
\text { Weekly }\end{array}$ & $\begin{array}{c}\text { Total } \\
\text { Session }\end{array}$ & $\begin{array}{l}\text { Outcome } \\
\text { Measured }\end{array}$ & $\begin{array}{c}\text { Blinded } \\
\text { Assessor }\end{array}$ & \\
\hline $\begin{array}{l}\text { Liu et al. } \\
{[49], \text { RCT }}\end{array}$ & $\begin{array}{l}\text { Changsha, China } \\
\text { (Chinese) }\end{array}$ & $40(20 / 20)$ & $0 \%$ & $\begin{array}{l}\text { People with Type } 2 \text { diabetic } \\
\text { mellitus }\end{array}$ & A mean age of 56 & $\begin{array}{c}\text { BJ: } 5 \times 30 \mathrm{~min} / \text { week for } 6 \text { months }+ \text { drug } \\
\text { therapy (as same below) } \\
\text { CG: drug therapy }\end{array}$ & 60 & 2.5 & 120 & $\begin{array}{c}\text { Pain } \\
\text { (subscale of } \\
\text { SAS) }\end{array}$ & NR & No/No \\
\hline $\begin{array}{l}\text { Wang, Liu et } \\
\text { al. [50], RCT }\end{array}$ & $\begin{array}{l}\text { Changsha, China } \\
\text { (Chinese) }\end{array}$ & $60(30 / 30)$ & $0 \%$ & $\begin{array}{l}\text { People with scapulohumeral } \\
\text { periarthritis }\end{array}$ & $\begin{array}{l}\text { A mean age } \\
\text { of } 53.54\end{array}$ & $\begin{array}{l}\text { BJ: } 7 \times 60 \mathrm{~min} / \text { week for } 30 \text { days }+ \\
\text { acupuncture (as same below) } \\
\text { CG: acupuncture therapy }\end{array}$ & 30 & 7 & 60 & Pain (NR) & NR & No/No \\
\hline $\begin{array}{l}\text { Xiao et al. } \\
{[51], \text { RCT }}\end{array}$ & $\begin{array}{l}\text { Shanghai, China } \\
\text { (Chinese) }\end{array}$ & $44(26 / 18)$ & $0 \%$ & $\begin{array}{c}\text { patients with cervical } \\
\text { spondylotic } \\
\text { radiculopathy/chronic neck } \\
\text { pain }\end{array}$ & A mean age of 51 & $\begin{array}{c}\text { BJ: } 7 \times 60 \mathrm{~min} / \text { week for } 30 \text { days }+ \text { drug } \\
\text { therapy } \\
\text { CG: drug therapy }\end{array}$ & 30 & 7 & 30 & Pain (VAS) & NR & No/No \\
\hline $\begin{array}{l}\text { Ding et al. } \\
\text { [52], RCT }\end{array}$ & $\begin{array}{l}\text { Hefei, China } \\
\text { (Chinese) }\end{array}$ & $40(22 / 18)$ & $0 \%$ & $\begin{array}{l}\text { people with chronic low back } \\
\text { pain }\end{array}$ & $\begin{array}{l}\text { A mean age } \\
\text { of } 60.98\end{array}$ & $\begin{array}{l}\text { BJ: } 5 \times 40 \mathrm{~min} / \text { week for } 12 \text { weeks } \\
\text { CG: usual drug therapy (pain killers) }\end{array}$ & 40 & 3.33 & 60 & Pain (VAS) & NR & No/No \\
\hline $\begin{array}{l}\text { Shang } \\
{[53], \text { RCT }}\end{array}$ & $\begin{array}{l}\text { Changchun, } \\
\text { China } \\
\text { (Chinese) }\end{array}$ & $60(30 / 30)$ & $0 \%$ & $\begin{array}{l}\text { People with lumbar disc } \\
\text { herniation }\end{array}$ & $\begin{array}{l}\text { Age range from } \\
18 \text { to } 60\end{array}$ & $\begin{array}{c}\text { BJ: } 5 \times 60 \text { min } / \text { week for } 3 \text { months + } \\
\text { usual care } \\
\text { CG: usual care }\end{array}$ & 60 & 5 & 60 & $\begin{array}{l}\text { Pain (VAS } \\
\text { and } \\
\text { JOABPEQ) }\end{array}$ & NR & No/No \\
\hline $\begin{array}{l}\text { Xuan et al. } \\
{[54], \text { RCT }}\end{array}$ & $\begin{array}{l}\text { Shanghai, China, } \\
\text { (Chinese) }\end{array}$ & $80(40 / 40)$ & $0 \%$ & $\begin{array}{l}\text { People with cervical } \\
\text { spondylotic radiculopathy } \\
\text { suffered from chronic neck } \\
\text { pain }\end{array}$ & $\begin{array}{l}\text { A mean age } \\
\text { of } 31.07\end{array}$ & $\begin{array}{l}\text { BJ: daily } 40 \text { min for } 20 \text { days + manual } \\
\text { therapy (as same below) } \\
\text { CG: manual therapy }\end{array}$ & 13 & 4.67 & 20 & $\begin{array}{c}\text { Pain } \\
\text { (SF-MPQ) }\end{array}$ & NR & No/No \\
\hline $\begin{array}{l}\text { Peng et al. } \\
\text { [55], RCT }\end{array}$ & $\begin{array}{l}\text { Guangzhou, } \\
\text { China } \\
\text { (Chinese) }\end{array}$ & $100(50 / 50)$ & $9 \%$ & $\begin{array}{l}\text { Older people with low back } \\
\text { pain who suffered from } \\
\text { osteoporosis }\end{array}$ & $\begin{array}{l}\text { a mean age } \\
\text { of } 69.06\end{array}$ & $\begin{array}{l}\text { BJ: daily } 30 \text { min for } 14 \text { days + usual care } \\
\text { CG: usual care }\end{array}$ & 7 & 3.5 & 14 & Pain (VAS) & NR & No/No \\
\hline $\begin{array}{l}\text { An et al. } \\
{[56], \text { RCT }}\end{array}$ & $\begin{array}{l}\text { Shanghai, China, } \\
\text { (English) }\end{array}$ & $28(14 / 14)$ & $25 \%$ & $\begin{array}{l}\text { Female patients with knee } \\
\text { osteoarthritis who suffered } \\
\text { from bodily pain }\end{array}$ & $\begin{array}{l}\text { a mean age } \\
\text { of } 65.0\end{array}$ & $\begin{array}{l}\text { BJ: } 5 \times 30 \mathrm{~min} / \text { week for } 8 \text { weeks } \\
\text { CG: no intervention }\end{array}$ & 20 & 2.5 & 40 & $\begin{array}{l}\text { Pain } \\
\text { (subscale of } \\
\text { WOMAC) }\end{array}$ & NR & NR/No \\
\hline $\begin{array}{l}\text { Wang et al. } \\
\text { [57] NRCT }\end{array}$ & $\begin{array}{l}\text { Qingdao, China, } \\
\text { (English) }\end{array}$ & $72(36 / 36)$ & $4.2 \%$ & $\begin{array}{l}\text { Patients with chronic neck } \\
\text { pain }\end{array}$ & $\begin{array}{l}\text { Age range } 45 \\
\text { to } 75\end{array}$ & $\begin{array}{c}\text { BJ: } 7 \times 30 \text { min } / \text { week for } 6 \text { months + } \\
\text { educational lessons } \\
\text { CG: educational lessons }\end{array}$ & 84 & 3.5 & 168 & Pain (VAS) & NR & NR/No \\
\hline
\end{tabular}


Table 1. Cont.

\begin{tabular}{|c|c|c|c|c|c|c|c|c|c|c|c|c|}
\hline \multirow{2}{*}{$\begin{array}{c}\text { Author } \\
\text { [Reference] }\end{array}$} & \multirow{2}{*}{$\begin{array}{l}\text { Location, } \\
\text { Language }\end{array}$} & \multirow[b]{2}{*}{ ISZ (BJ/CG) } & \multirow[b]{2}{*}{ Drop-Out $\%$} & \multicolumn{2}{|c|}{ Study Participants } & \multicolumn{4}{|c|}{ Intervention Protocol } & \multicolumn{2}{|c|}{ Test Administration } & \multirow{2}{*}{$\begin{array}{l}\text { Adverse Event; } \\
\text { Follow-Up }\end{array}$} \\
\hline & & & & Health Status & $\begin{array}{l}\text { Mean Age or } \\
\text { Age Range }\end{array}$ & Training Duration and Dosage & $\begin{array}{c}\text { Total } \\
\text { Hours }\end{array}$ & $\begin{array}{c}\text { Hours } \\
\text { Weekly }\end{array}$ & $\begin{array}{l}\text { Total } \\
\text { Session }\end{array}$ & $\begin{array}{l}\text { Outcome } \\
\text { Measured }\end{array}$ & $\begin{array}{c}\text { Blinded } \\
\text { Assessor }\end{array}$ & \\
\hline $\begin{array}{l}\text { Xiao et al. } \\
\text { [58], RCT }\end{array}$ & $\begin{array}{l}\text { Beijing, China } \\
\text { (English) }\end{array}$ & $96(48 / 48)$ & $7.3 \%$ & $\begin{array}{l}\text { Patients with Parkinson's } \\
\text { disease }\end{array}$ & $\begin{array}{l}\text { Age range from } \\
55 \text { to } 80\end{array}$ & $\begin{array}{c}\text { BJ: } 4 \times 45 \text { min/week for } 6 \text { months + daily } \\
\text { walking for } 30 \mathrm{~min} \\
\text { CG: daily walking for } 30 \mathrm{~min}\end{array}$ & 72 & 3 & 96 & $\begin{array}{l}\text { Sleep quality } \\
\text { (PDSS-2) }\end{array}$ & NR & No/No \\
\hline $\begin{array}{l}\text { Chan et al. } \\
\text { [59], RCT }\end{array}$ & $\begin{array}{l}\text { Hong Kong, } \\
\text { China } \\
\text { (English) }\end{array}$ & $150(75 / 75)$ & $13.3 \%$ & $\begin{array}{l}\text { People with chronic fatigue } \\
\text { syndrome-like illness }\end{array}$ & $\begin{array}{l}\text { A mean age } \\
\text { of } 39.1\end{array}$ & $\begin{array}{c}\text { BJ: } 90 \text { mn per session over } 9 \text { consecutive } \\
\text { weeks, for } 16 \text { sessions } \\
\text { CG: waitlist } \\
\end{array}$ & 24 & 2.6 & 16 & $\begin{array}{l}\text { Sleep quality } \\
\text { (PSQI) }\end{array}$ & NR & No/3-month \\
\hline $\begin{array}{l}\text { Wang et al. } \\
\text { [60], RCT }\end{array}$ & $\begin{array}{l}\text { Beijing, China } \\
\text { (Chinese) }\end{array}$ & $\begin{array}{c}90 \\
(30 / 30 / 30)\end{array}$ & $13.3 \%$ & $\begin{array}{l}\text { Patients with type } 2 \text { Diabetic } \\
\text { mellitus accompanied by } \\
\text { insomnia }\end{array}$ & $\begin{array}{l}\text { a mean age } \\
\text { of } 57.8\end{array}$ & $\begin{array}{l}\text { BJ: training dosage was NR for } 4 \text { months } \\
\text { + usual care } \\
\text { CG1: LiuZijue (Training dosage was NR) } \\
\text { for } 4 \text { months + usual care } \\
\text { CG2: usual care }\end{array}$ & $\mathrm{NA}$ & $\mathrm{NA}$ & $\mathrm{NA}$ & $\begin{array}{l}\text { Sleep quality } \\
\text { (PSQI) }\end{array}$ & NR & No/No \\
\hline $\begin{array}{l}\text { Xiong et al. } \\
\text { [61], RCT }\end{array}$ & $\begin{array}{l}\text { Changchun, } \\
\text { China, } \\
\text { (Chinese) }\end{array}$ & $\begin{array}{c}60 \\
(20 / 20 / 20)\end{array}$ & $0 \%$ & $\begin{array}{l}\text { Middle-aged adults with } \\
\text { insomnia }\end{array}$ & $\begin{array}{l}\text { A mean age } \\
\text { of } 48.7\end{array}$ & $\begin{array}{l}\text { BJ1: } 5 \times 40 \mathrm{~min} / \text { week for } 4 \text { weeks } \\
\text { BJ2: Baduanjin (same dosage as above) + } \\
\text { acupuncture } \\
\text { CG: acupuncture }\end{array}$ & 13.3 & 3.3 & 20 & $\begin{array}{l}\text { Sleep quality } \\
\text { (PSQI) }\end{array}$ & NR & No/No \\
\hline $\begin{array}{l}\text { Chen et al. } \\
\text { [62], NRCT }\end{array}$ & $\begin{array}{l}\text { Fuzhou, China, } \\
\text { (Chinese) }\end{array}$ & $60(30 / 30)$ & $0 \%$ & $\begin{array}{l}\text { Older people with } \\
\text { hypertension accompanied by } \\
\text { insomnia }\end{array}$ & $\begin{array}{l}\text { Age range from } \\
\quad 60 \text { to } 75\end{array}$ & $\begin{array}{l}\text { BJ: } 3 \times 60 \mathrm{~min} / \text { week for } 3 \text { months }+ \\
\text { educational lessons } \\
\text { CG: educational lessons }\end{array}$ & 36 & 3 & 36 & $\begin{array}{c}\text { Sleep quality } \\
\text { (PSQI) }\end{array}$ & NR & $\mathrm{No} / \mathrm{No}$ \\
\hline $\begin{array}{l}\text { Li et al. } \\
\text { [63], RCT }\end{array}$ & $\begin{array}{l}\text { Jiangsu, China, } \\
\text { (Chinese) }\end{array}$ & $40(20 / 20)$ & $0 \%$ & $\begin{array}{l}\text { Patients with type } 2 \text { Diabetic } \\
\text { mellitus accompanied by } \\
\text { insomnia (mean age of } 53.6 \text { ) }\end{array}$ & $\begin{array}{l}\text { A mean age } \\
\text { of } 53.6\end{array}$ & $\begin{array}{l}\text { BJ: } 7 \times 30 \mathrm{~min} / \text { week for } 4 \text { weeks + } \\
\text { educational lessons } \\
\text { CG: educational lessons }\end{array}$ & 14 & 5.17 & 28 & $\begin{array}{l}\text { Sleep quality } \\
\text { (PSQI) }\end{array}$ & NR & No/No \\
\hline $\begin{array}{l}\text { Xiong et al. } \\
\text { [64], RCT }\end{array}$ & $\begin{array}{l}\text { Changchun, } \\
\text { China (Chinese) }\end{array}$ & $60(30 / 30)$ & $0 \%$ & People with insomnia & $\begin{array}{l}\text { Age range from } \\
18 \text { to } 75\end{array}$ & $\begin{array}{l}\text { BJ1: } 5 \times 40 \mathrm{~min} / \text { week for } 4 \text { weeks } \\
\text { BJ2: Baduanjin (same dosage as above) + } \\
\text { acupuncture } \\
\text { CG: acupuncture }\end{array}$ & 13.3 & 3.3 & 20 & $\begin{array}{l}\text { Sleep quality } \\
\text { (PSQI) }\end{array}$ & NR & No/No \\
\hline \multicolumn{13}{|c|}{ Pretest-posttest study without control group } \\
\hline \multirow{2}{*}{$\begin{array}{l}\text { Author } \\
\text { Reference }\end{array}$} & \multirow{2}{*}{$\begin{array}{l}\text { Study location } \\
\text { (Language) }\end{array}$} & \multirow{2}{*}{$\begin{array}{c}\text { Initial } \\
\text { sample size }\end{array}$} & \multirow{2}{*}{ Drop-Out \% } & \multicolumn{2}{|c|}{ Study characteristic } & \multicolumn{4}{|c|}{ Intervention protocol } & \multicolumn{3}{|c|}{ Study findings } \\
\hline & & & & Health status & $\begin{array}{l}\text { Age range or } \\
\text { mean age }\end{array}$ & Training Duration and Dosage & $\begin{array}{l}\text { Total } \\
\text { Hours }\end{array}$ & $\begin{array}{c}\text { Hours } \\
\text { Weekly }\end{array}$ & $\begin{array}{c}\text { Total } \\
\text { Session }\end{array}$ & $\begin{array}{l}\text { Outcome } \\
\text { Measured }\end{array}$ & Stuc & y results \\
\hline $\begin{array}{l}\text { An et al. } \\
{[65], \text { PPS }}\end{array}$ & $\begin{array}{l}\text { Shanghai, China, } \\
\text { (English) }\end{array}$ & 28 & $21.4 \%$ & $\begin{array}{l}\text { Patients with knee } \\
\text { osteoarthritis }\end{array}$ & $\begin{array}{l}\text { A mean age } \\
\text { of } 65.2\end{array}$ & BJ: $5 \times 30 \mathrm{~min} /$ week for 1 year & 120 & 2.5 & 336 & $\begin{array}{l}\text { Pain } \\
\text { (subscale of } \\
\text { WOMAC) }\end{array}$ & $\begin{array}{l}\text { BJ significa } \\
\text { level of pa } \\
\text { vs. } 56.2 \pm\end{array}$ & $\begin{array}{l}\text { ntly reduced the } \\
\text { in }(132.0 \pm 69.6 \\
67.6, p=0.000)\end{array}$ \\
\hline
\end{tabular}


Table 2. Methodological quality for randomized controlled trials and non-randomized controlled studies.

\begin{tabular}{|c|c|c|c|c|c|c|c|c|c|c|c|c|}
\hline Author (Reference) & Item 1 & Item 2 & Item 3 & Item 4 & Item 5 & Item 6 & Item 7 & Item 8 & Item 9 & Item 10 & Item 11 & Score \\
\hline Du et al. [37] & 1 & 1 & 0 & 1 & 0 & 1 & 1 & 1 & 1 & 0 & 0 & $6 / 10$ \\
\hline Wang et al. [38] & 1 & 1 & 0 & 1 & 0 & 1 & 0 & 1 & 1 & 0 & 0 & $5 / 10$ \\
\hline Cai et al. [39] & 1 & 1 & 0 & 1 & 0 & 1 & 1 & 1 & 1 & 0 & 0 & $6 / 10$ \\
\hline Li et al. [40] & 1 & 1 & 0 & 1 & 0 & 1 & 1 & 1 & 1 & 0 & 0 & $6 / 10$ \\
\hline Xu et al. [41] & 1 & 1 & 0 & 1 & 0 & 1 & 1 & 0 & 1 & 0 & 0 & $5 / 10$ \\
\hline Chen et al. [42] & 1 & 1 & 0 & 1 & 0 & 1 & 1 & 1 & 1 & 0 & 0 & $6 / 10$ \\
\hline Wei et al. [44] & 1 & 1 & 0 & 1 & 0 & 1 & 1 & 1 & 1 & 0 & 0 & $6 / 10$ \\
\hline Wan et al. [45] & 1 & 1 & 0 & 1 & 0 & 1 & 1 & 1 & 1 & 0 & 0 & $6 / 10$ \\
\hline Wang et al. [46] & 1 & 1 & 0 & 1 & 0 & 1 & 0 & 1 & 1 & 0 & 0 & $5 / 10$ \\
\hline Fu et al. [47] & 1 & 1 & 0 & 1 & 0 & 1 & 1 & 1 & 1 & 0 & 0 & $6 / 10$ \\
\hline Li et al. [48] & 1 & 1 & 0 & 1 & 0 & 1 & 1 & 1 & 1 & 0 & 0 & $6 / 10$ \\
\hline Liu et al. [49] & 1 & 1 & 0 & 1 & 0 & 1 & 1 & 1 & 1 & 0 & 0 & $6 / 10$ \\
\hline Wang \& Liu [50] & 1 & 1 & 0 & 1 & 0 & 1 & 1 & 1 & 1 & 0 & 0 & $6 / 10$ \\
\hline Xiao et al. [51] & 1 & 1 & 0 & 1 & 0 & 1 & 1 & 1 & 1 & 0 & 0 & $6 / 10$ \\
\hline Ding et al. [52] & 1 & 1 & 0 & 1 & 0 & 1 & 1 & 1 & 1 & 1 & 0 & $7 / 10$ \\
\hline Shang [53] & 1 & 1 & 0 & 1 & 0 & 1 & 1 & 1 & 1 & 0 & 0 & $6 / 10$ \\
\hline Xuan et al. [54] & 1 & 1 & 0 & 1 & 0 & 1 & 1 & 1 & 1 & 0 & 0 & $6 / 10$ \\
\hline Peng et al. [55] & 1 & 1 & 0 & 1 & 0 & 1 & 0 & 1 & 1 & 0 & 0 & $5 / 10$ \\
\hline An et al. [56] & 1 & 1 & 0 & 1 & 0 & 0 & 0 & 1 & 1 & 1 & 0 & $5 / 10$ \\
\hline Wang et al. [57] & 1 & 0 & 0 & 1 & 0 & 1 & 0 & 1 & 1 & 0 & 0 & $4 / 10$ \\
\hline Xiao et al. [58] & 1 & 1 & 0 & 1 & 0 & 1 & 1 & 1 & 1 & 0 & 0 & $6 / 10$ \\
\hline Chan et al. [59] & 1 & 1 & 0 & 1 & 0 & 1 & 1 & 1 & 1 & 1 & 1 & $8 / 10$ \\
\hline Wang et al. [60] & 1 & 1 & 0 & 1 & 0 & 1 & 1 & 1 & 1 & 0 & 0 & $6 / 10$ \\
\hline Xiong et al. [61] & 1 & 1 & 0 & 1 & 0 & 1 & 1 & 1 & 1 & 0 & 0 & $6 / 10$ \\
\hline Chen et al. [62] & 1 & 0 & 0 & 1 & 0 & 1 & 1 & 1 & 1 & 0 & 0 & $5 / 10$ \\
\hline Li et al. [63] & 1 & 1 & 0 & 1 & 0 & 1 & 1 & 0 & 1 & 0 & 0 & $5 / 10$ \\
\hline Xiong et al. [64] & 1 & 1 & 0 & 1 & 0 & 1 & 1 & 1 & 1 & 0 & 0 & $6 / 10$ \\
\hline
\end{tabular}

Note: Item 1 = eligibility criteria (does not contribute to total score); Item $2=$ randomization; Item $3=$ concealed allocation; Item $4=$ similar baseline; Item $5=$ blinding of assessors; Item $6=$ more than $85 \%$ retention; Item $7=$ missing data management (intention-to-treat analysis); Item $8=$ between-group comparison; Item $9=$ point measure and measures of variability; Item $10=$ isolated Baduanjin intervention; Item 11 = prior sample size estimation; 1 = explicitly described and present in details; $0=$ absent, inadequately described, or unclear 


\subsection{Effects of Baduanjin on Musculoskeletal Pain and Overall Sleep Quality}

\subsubsection{Baduanjin Intervention versus Control Group on Musculoskeletal Pain}

There were two types of testing instrument conditions for measuring musculoskeletal pain: (1) higher scores indicate more severe musculoskeletal pain (e.g., VAS and the SF-MPQ) as Condition 1; (2) higher scores indicate less pain (e.g., JOABPEQ) as Condition 2. For Condition one, there were 18 different studies (19 comparisons because one study by Wan et al. [45] used both VAS and SF-MPQ). The funnel plot was used to visually determine if the outliers existed: two studies $(\mathrm{SMD}=-2.75$ and $\mathrm{SMD}=-2.37)$ reported large effect sizes beyond $2 \mathrm{SD}$ above the mean effect size of -1.05 (random-effect model) [40,59]. While these two outliers were removed for further analysis, the funnel plot of remaining studies show no significant asymmetry (Egger's regression intercept $=-0.28$, $p=0.85$ ). For the mete-analysis, the 16 remaining studies (17 pairs of comparisons from 15 RCTs and 1 NRCT) examined the effects of Baduanjin versus control group on musculoskeletal pain. A higher negative value (effect size) indicates greater alleviation of musculoskeletal pain. The aggregated result has shown a significant benefit in favour of Baduanjin on alleviating musculoskeletal pain (a large effect size, but low heterogeneity: SMD $=-0.88,95 \% \mathrm{CI}-1.02$ to $-0.74, p<0.001, I^{2}=10.29 \%$; Figure 2). In the meta-analysis in Condition 2, there were three studies (4 pairs of comparisons); a higher positive value indicates greater alleviation of musculoskeletal pain. The aggregated result has shown a significant benefit in favour of Baduanjin on alleviating musculoskeletal pain (a large effect size without heterogeneity: $\mathrm{SMD}=0.87,95 \%$ CI 0.58 to $1.16, p<0.001, I^{2}=0 \%$; Figure 3 ).

\section{Musculoskeletal Pain:Baduanjin vs Control}

\begin{tabular}{|c|c|c|c|c|}
\hline \multirow[t]{2}{*}{ Study name } & \multicolumn{4}{|c|}{ Statistics for each study } \\
\hline & \multicolumn{4}{|c|}{$\begin{array}{l}\text { Std diff LowerUpper } \\
\text { in means limit limit } p\end{array}$} \\
\hline Du et al [37] & -0.83 & -1.42 & -0.24 & 0.01 \\
\hline Wang et al [38] & -0.98 & -1.47 & -0.49 & 0.00 \\
\hline Cai et al [39] & -0.42 & -0.93 & 0.10 & 0.11 \\
\hline Xu et al. [41] & -0.47 & -1.48 & 0.52 & 0.35 \\
\hline Wei et al. [44] & -1.38 & -1.93 & -0.83 & 0.00 \\
\hline Wan et al [45]VAS & -0.65 & -1.17 & -0.14 & 0.01 \\
\hline \multicolumn{2}{|c|}{ Wan et al [45]SF-MPO-D.55 } & -1.06 & -0.03 & 0.04 \\
\hline Wang et al [46] & -0.77 & -1.27 & -0.26 & 0.00 \\
\hline Fu et $s \mid[47]$ & -1.04 & -1.54 & -0.55 & 0.00 \\
\hline Li et al [48] & -1.14 & -1.88 & -0.59 & 0.00 \\
\hline Liu et al [49] & -1.03 & -1.69 & -0.37 & 0.00 \\
\hline Xiso et al [51] & -0.88 & -1.51 & -0.25 & 0.01 \\
\hline Ding et al [52] & -1.39 & -2.09 & -0.70 & 0.00 \\
\hline Shang [53] & -1.32 & -1.88 & -0.76 & 0.00 \\
\hline Xuan et al [54] & -0.92 & -1.38 & -0.48 & 0.00 \\
\hline An et al [58] & -0.80 & -1.48 & 0.27 & 0.18 \\
\hline \multirow[t]{2}{*}{ Wang et al [57] } & -0.55 & -1.03 & -0.07 & 0.03 \\
\hline & -0.88 & -1.02 & -0.74 & 0.00 \\
\hline
\end{tabular}

Std diff in means and $95 \% \mathrm{CI}$

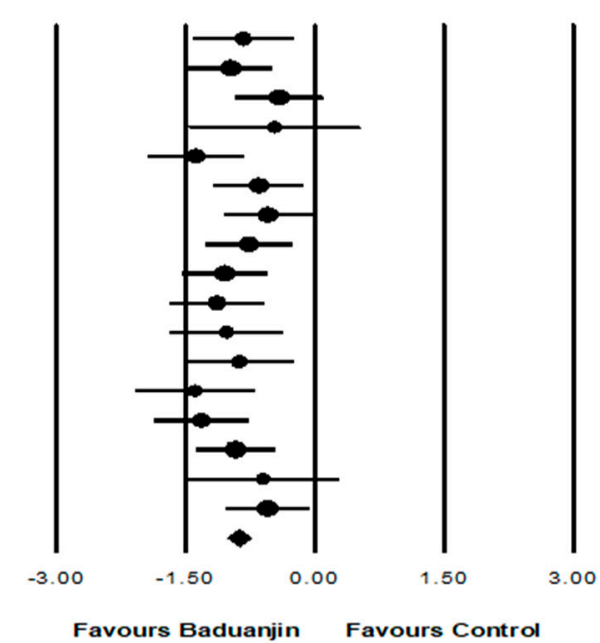

Figure 2. Effect of Baduanjin on musculoskeletal pain (Condition 1). 


\section{Musculoskeletal Pain:Baduanjin vs Control}

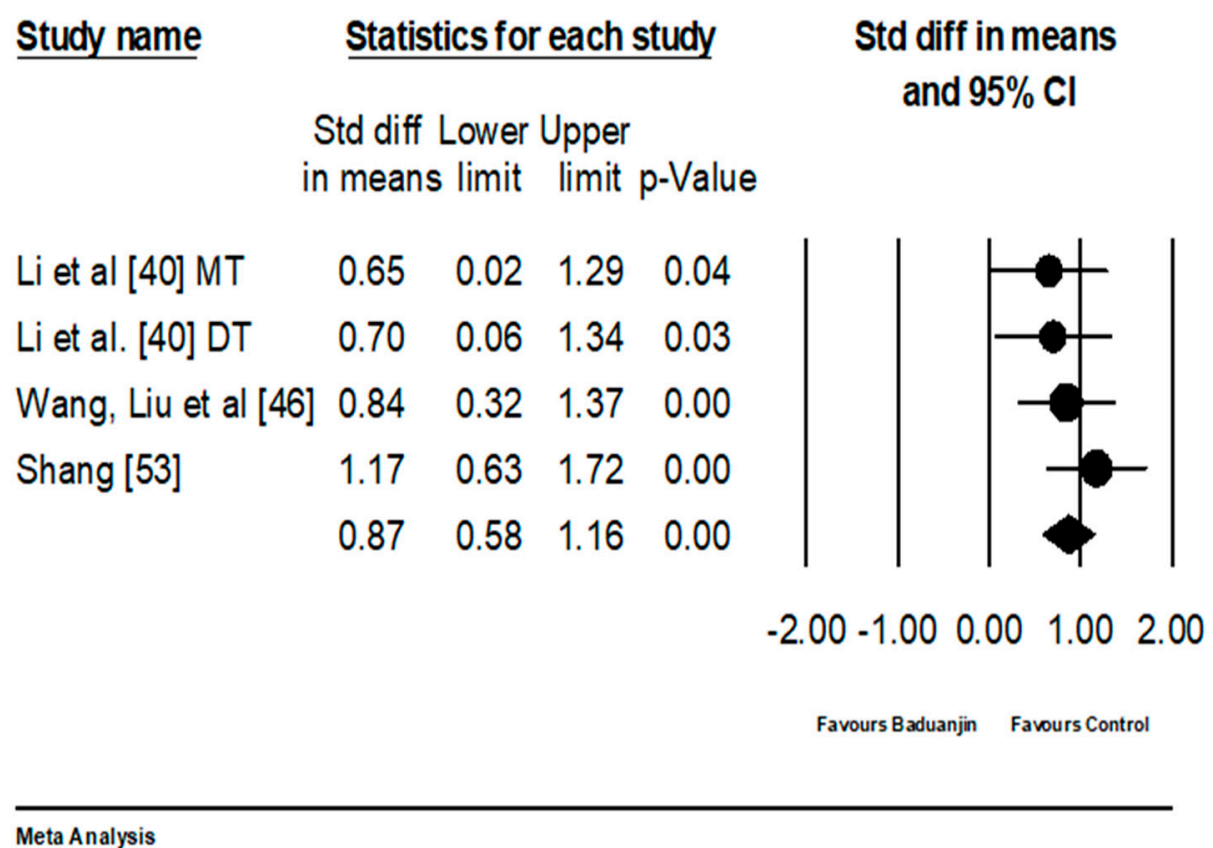

Figure 3. Effect of Baduanjin on musculoskeletal pain (Condition 2); MT = manual therapy; DT = drug therapy).

\subsubsection{Baduanjin Intervention versus Control Group on Overall Sleep Quality}

There were 7 studies ( 6 RCTs and 1 NRCT) investigating the effects of Baduanjin on overall sleep quality. Because three studies involved two control groups, ten pairs of comparisons were included for the meta-analysis $[46,61,64]$. A higher negative value (effect size) indicates better overall sleep quality. The aggregated result has shown a significant benefit in favour of Baduanjin on overall sleep quality (a large effect size, but high heterogeneity: SMD $=-0.84,95 \% \mathrm{CI}-1.38$ to -0.29 , $p=0.002, I^{2}=89.31 \%$; Figure 4$)$. The funnel plot was used to visually determine if the outliers existed: two pairs of comparisons $(\mathrm{SMD}=-2.52$ and $\mathrm{SMD}=-2.34$ ) reported large effect sizes beyond $2 \mathrm{SD}$ above the mean effect size was -0.83 (random-effect model). While these two outliers were removed for further analysis, the funnel plot of remaining studies showing no significant asymmetry (Egger's regression intercept $=-0.376, p=0.927)$. For the mete-analysis, the remaining comparisons examined the effects of Baduanjin versus control group on overall sleep quality. A higher negative value (effect size) indicates better overall sleep quality. The aggregated result has shown a significant benefit in favour of Baduanjin on alleviating overall sleep quality (a moderate effect size, but high heterogeneity: $\mathrm{SMD}=-0.48,95 \% \mathrm{CI}-0.95$ to $-0.01, p=004, I^{2}=84.42 \%$; Figure 5 ). 


\section{Overall Sleep Quality: Baduanjin vs Control}

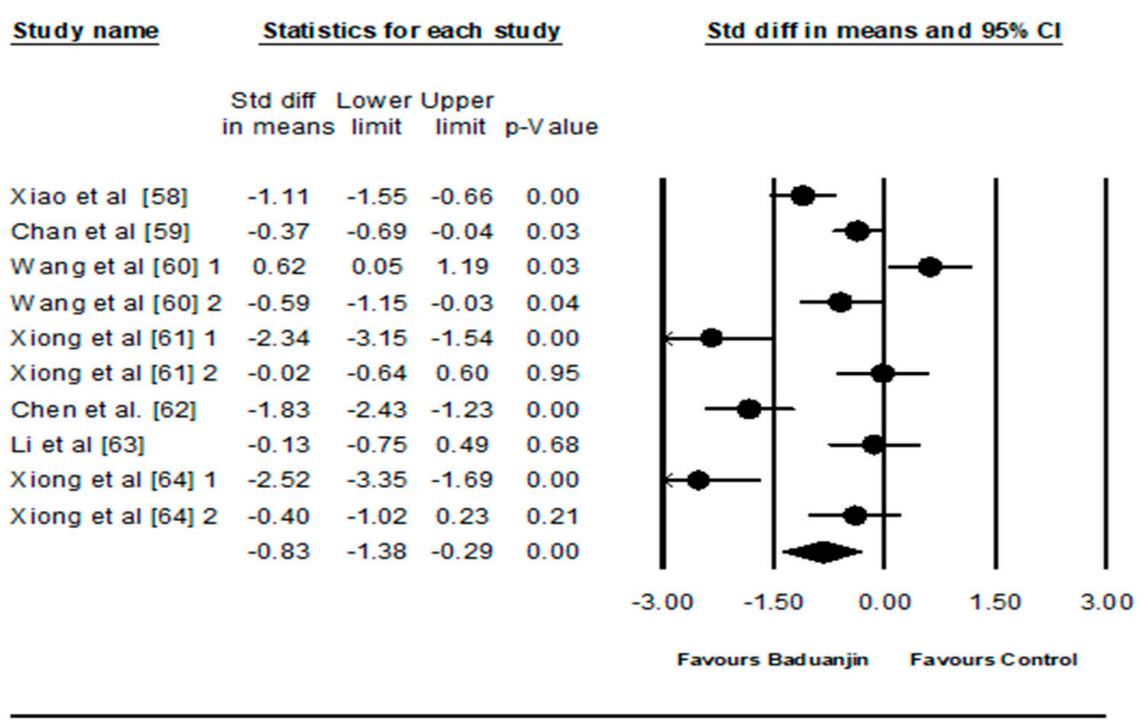

Meta Analysis

Figure 4. Effect of Baduanjin on overall sleep quality across all studies selected; Wang et al. [60] 1 = Baduanjin vs. LiuZijue; Wang et al. [60] 2 = Baduanjin vs. no training; Xiong et al. [61] 1 = Baduanjin + acupuncture vs. acupuncture; Xiong et al. [61] 2 = Baduanjin vs. acupuncture; Xiong et al. [64] 1 = Baduanjin + acupuncture vs. acupuncture; Xiong et al. [64] $2=$ Baduanjin vs. acupuncture). 1 is referring to the first comparison, which is Baduanjin vs. Liuzijue; 2 for the second comparison in the same study.

\section{Overall Sleep Quality: Baduanjin vs Control}

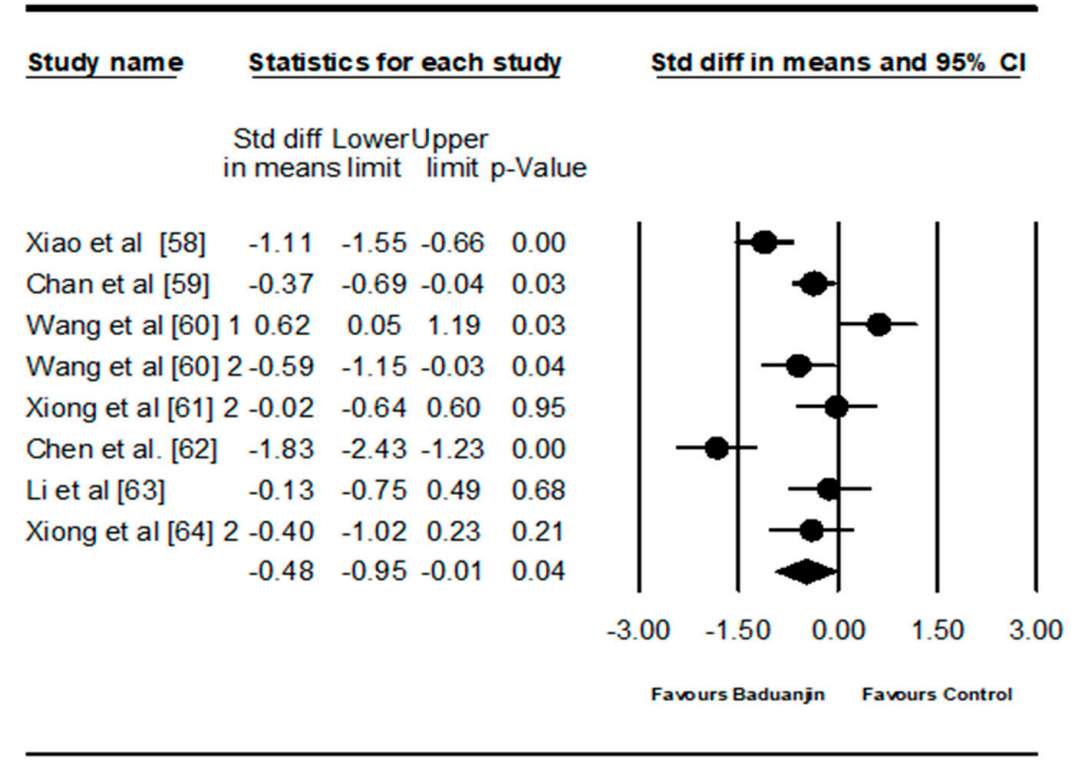

Meta Analysis

Figure 5. Effect of Baduanjin on overall sleep quality (without including two outliers); Wang et al. [60] 1 = Baduanjin vs. LiuZijue; Wang et al. [60] 2 = Baduanjin vs. no training; Xiong et al. [61] 2 = Baduanjin vs. acupuncture; Xiong et al. [64] 2 = Baduanjin vs. acupuncture). 1 is referring to the first comparison, which is Baduanjin vs. Liuzijue; 2 for the second comparison in the same study. 


\subsubsection{Moderator Analysis}

The effects of potential moderator variables were computed only for musculoskeletal pain and not for overall sleep quality (the number of studies was less than 10). The results of categorical and continuous moderator analysis are presented in Table 3. With regard to the musculoskeletal pain, because the specific intervention length was not reported in five studies $[45,50,51,54,55]$, only 13 studies were considered for subgroup analysis on the intervention length. With respect to the subgroup analysis for the rest of the categorical variables (final sample size, weekly training hours, sessions length, and control type), the 16 remaining studies (17 pairs of comparisons) were included. There were no significant effects for intervention length $(Q(1)=0.19, p=0.66)$, final sample size $(Q(1)=0.07$, $p=0.76)$, weekly training hours $(Q(1)=0.49, p=0.49)$, session length $(Q(1)=0.49, p=0.49)$, and type of control group $(Q(1)=0.21, p=0.65)$. With regard to depression, there was a marginally insignificant effect for type of control group $(Q(1)=3.74, p=0.053)$, whereas no significant effect was observed for intervention duration $(Q(1)=0.03, p=0.87)$, training frequency $(Q(1)=2.8, p=0.09)$, session length $(Q(1)=0.7, p=0.4)$, and study quality $(Q(1)=0.44, p=0.51)$. For continuous potential moderators, meta-regression indicated no significant effects for total hours in Baduanjin practice $(\beta=-0.00111$, $95 \% \mathrm{CI}-0.0017$ to $-0.0039, p=0.44)$ and the number of total sessions $(\beta=-0.00108,95 \% \mathrm{CI}-0.00136$ to $0.00352, p=0.39$ ) in terms of musculoskeletal pain. 
Table 3. Moderator analysis for Baduanjin versus control group.

\begin{tabular}{|c|c|c|c|c|c|c|c|c|c|}
\hline \multirow{2}{*}{ Categorical Moderator } & \multirow{2}{*}{ Outcome } & \multirow{2}{*}{ Level } & \multirow{2}{*}{$\begin{array}{c}\text { No. of } \\
\text { Studies/Comparisons }\end{array}$} & \multirow{2}{*}{ SMD } & \multirow{2}{*}{ 95\% Confidence Interval } & \multirow{2}{*}{$I^{2}, \%$} & \multicolumn{3}{|c|}{ Test for between-Group Homogeneity } \\
\hline & & & & & & & $Q$-Value & $\mathrm{df}(Q)$ & $p$-Value \\
\hline \multirow[t]{2}{*}{ Intervention Length } & \multirow{2}{*}{ Pain } & $<12$ weeks & 4 & -0.85 & -1.24 to -0.46 & $0 \%$ & 0.19 & 1 & 0.66 \\
\hline & & $\geq 12$ weeks & 9 & -0.95 & -1.17 to -0.74 & $39.03 \%$ & & & \\
\hline \multirow{2}{*}{ Final sample size } & \multirow{2}{*}{ pain } & $<60$ & 6 & -0.92 & -1.22 to -0.61 & $0 \%$ & 0.07 & 1 & 0.79 \\
\hline & & $\geq 60$ & 11 & -0.87 & -1.04 to -0.70 & $30.78 \%$ & & & \\
\hline \multirow{2}{*}{ Weekly training hours } & \multirow{2}{*}{ Pain } & $<5 \mathrm{~h}$ per week & 7 & -0.95 & -1.18 to -0.71 & $18.7 \%$ & 0.49 & 1 & 0.49 \\
\hline & & $\geq 5$ h per week & 10 & -0.84 & -1.03 to -0.65 & $9.41 \%$ & & & \\
\hline \multirow{3}{*}{ Session length } & \multirow{3}{*}{ Pain } & Less than 45 & 7 & -0.95 & -1.18 to -0.71 & $18.7 \%$ & 0.49 & 1 & 0.49 \\
\hline & & $\begin{array}{c}\min \\
45 \mathrm{~min} \text { or }\end{array}$ & $\gamma$ & & & & & & \\
\hline & & $\begin{array}{l}45 \mathrm{~min} \text { or } \\
\text { longer }\end{array}$ & 10 & -0.84 & -1.03 to -0.65 & $9.41 \%$ & & & \\
\hline \multirow{2}{*}{ Control type } & \multirow{2}{*}{ Pain } & Active & 11 & -0.91 & -1.09 to -0.72 & $12.78 \%$ & 0.21 & 1 & 0.65 \\
\hline & & passive & 6 & -0.83 & -1.08 to -0.59 & $18.63 \%$ & & & \\
\hline Continuous moderator & & Level & $\begin{array}{c}\text { No. of } \\
\text { Studies/Comparisons }\end{array}$ & $\beta$ & 95\% Confidence Interval & & $Q$-Value & $\mathrm{df}(Q)$ & $p$-Value \\
\hline Total training hours & & Pain & 17 & -0.00111 & -0.0017 to 0.0039 & & 0.6 & 1 & 0.44 \\
\hline Number of total sessions & & Pain & 17 & -0.00108 & -0.00136 to 0.00352 & & 0.75 & 1 & 0.39 \\
\hline
\end{tabular}




\section{Discussion}

This systematic review critically evaluated and statistically synthesized the evidence of the effects of Baduanjin exercise on musculoskeletal pain and sleep quality. Based on the available evidence, our review suggests that this traditional mind body exercise reduces musculoskeletal pain and improves sleep quality among patients with chronic illnesses. This is the first meta-analysis of the effects of Baduanjin on pain and sleep of patients with chronic illnesses. The data from the emerging literature provide support to Baduanjin as a self-management practice to augment conventional treatments for those with chronic illnesses. While the exact mechanisms of how Baduanjin affects musculoskeletal pain and sleep are unknown, the findings from this study are of great public health significance, since musculoskeletal pain and sleep disturbance are highly prevalent among these patients, and tremendous medical resources are used to address these symptoms. Contemporary concept Qigong practices like Baduanjin enhance physiological proprioception by combining a special state of awareness with posture, movement, and breathe control, and thereby improve and strengthen the overall state of vegetative regulation (homeostasis) [66]. Compared to first line treatments (drugs, cognitive behavioral therapy) and other effective treatment alternatives (e.g., aerobic exercise for insomnia), there are a lot of advantage to use Baduanjin exercise as an adjunctive treatment for patients suffering from pain and from insomnia. It is accessible to people of all ages and physical strength, easy to learn, and has few known side effects. This is particularly important for elderly patients who are prone to medication side effects and potential drug-drug interactions. The disadvantages are that qualified Qigong instructors may not be available in many areas, and that long term adherence of self-management practices tends to be low.

This study included recently published empirical studies in both English and Chinese that used Baduanjin as the primary intervention. This method is appropriate and important, since, thus far, all of the studies on Baduanjin were conducted in China (including Hong Kong) and were published mostly in the Chinese language. In including articles in Chinese, the contributions of researchers on Baduanjin studies published in Chinese peer-review journals are acknowledged, and the findings are more representative of the studies in this area. Other strengths of this study include the use of a standardized scale to assess the methodological quality of the studies, and a recognized meta-analytic method to evaluate the magnitude of Baduanjin intervention effect (pooled effect size), the variations in the frequency and duration of Baduanjin practice (moderator analyses), and the extent of asymmetry of effect sizes (funnel plot and Egger's regression).

Nonetheless, the following methodological limitations could be acknowledged as they may influence the interpretation of these research findings. One of the most important drawbacks is that there was a lack of blinding in most studies, which might lead to subjectivity and social desirability bias. Second, Baduanjin was not offered as a monotherapy, but as an adjunctive treatment to the interventions received by the patients. It may be difficult to definitely conclude whether the outcomes were due to Baduanjin alone, to a synergetic intervention effect, or to the conventional treatment received by the patients. Nevertheless, our data provide support for Baduanjin as an adjunctive treatment for patients with chronic illnesses to reduce pain and improve sleep. Third, a variety of interventions were received by control groups, which made interpretations of outcomes difficult. Fourth, the duration of the Baduanjin practice varied a lot among different studies. This makes it hard to make specific recommendations about how frequent and how long practices should be. In addition, it is unclear why treatment effects did not differ by intervention length, frequency, and session length in moderator analysis. The intervention length of most studies was 4 weeks or more. It is possible that they have all attained the minimal duration needed to learn the Baduanjin postures and movements. The frequency and session length may be less important given that Baduanjin is relatively easy to learn, and that the majority of the benefits might have come from daily self-practice and not from the training sessions. Fifth, all the studies were conducted in China (mainland China and Hong Kong), and the participants were predominantly Chinese. It remains unclear whether the results are generalizable to non-Chinese populations. Sixth, a variety of heterogeneous chronic illnesses were included, consisting 
of insomnia, diabetes, hypertension, and various musculoskeletal conditions. It is not clear whether the beneficial effects of Baduanjin apply to all or only some of the chronic illnesses. Lastly, studies that report positive or significant results are more likely to be published, and outcomes that are statistically significant have greater possibility of being fully reported. Publication bias and outcome reporting bias might have existed in the included studies, and the effect sizes of Baduanjin might have been overestimated.

\section{Conclusions}

This systematic review, based on the existing literature, suggests that Bajuanjin may be an effective intervention to alleviate musculoskeletal pain and improve sleep quality among individuals with chronic illnesses. Significant methodological limitations were found in many of the empirical studies to date, which impacted the interpretation of these findings. More RCTs with rigorous research design are warranted to establish the therapeutic effects of Bajuanjin for musculoskeletal pain relief and improving sleep quality, and its potential to be used in part of healthy lifestyle intervention programs for populations with various clinical conditions.

Acknowledgments: This study was supported by Shanghai Philosophy and sociology (No. 2017BTY002); the fund for Science and Technology Innovation of Shanghai Jiao Tong University (No. JCZD005).

Author Contributions: Liye Zou, Albert Yeung, and Xinfeng Quan contributed to the conception and design of the review. Liye Zou and Xinfeng Quan. Applied the search strategy. All authors applied the selection criteria. All authors completed assessment of risk of bias. All authors analyzed the data and interpreted data. Liye Zou and Albert Yeung wrote this manuscript. Sean David Boyden and Huiru Wang edited this manuscript. Liye Zou is responsible for the overall project.

Conflicts of Interest: The authors declare no conflicts of interest.

\section{References}

1. Breivik, H.; Collett, B.; Ventafridda, V.; Cohen, R.; Gallacher, D. Survey of chronic pain in Europe: Prevalence, impact on daily life, and treatment. Eur. J. Pain 2006, 10, 287-333. [CrossRef] [PubMed]

2. Eriksen, J.; Jensen, M.K.; Sjøgren, P.; Ekholm, O.; Rasmussen, N.K. Epidemiology of chronic non-malignant pain in Denmark. Pain 2003, 106, 221-228. [CrossRef]

3. Rustøen, T.; Wahl, A.K.; Hanestad, B.R.; Lerdal, A.; Paul, S.; Miaskowski, C. Prevalence and characteristics of chronic pain in the general Norwegian population. Eur. J. Pain 2004, 8, 555-565. [CrossRef] [PubMed]

4. Ng, K.F.J.; Tsui, S.L.; Chan, W.S. Prevalence of common chronic pain in Hong Kong adults. Clin. J. Pain 2002, 18, 275-281. [CrossRef] [PubMed]

5. Yeo, S.N.; Tay, K.H. Pain prevalence in Singapore. Ann. Acad. Med. Singap. 2009, 38, 937-942. [PubMed]

6. Nakamura, M.; Nishiwaki, Y.; Ushida, T.; Toyama, Y. Prevalence and characteristics of chronic musculoskeletal pain in Japan. J. Orthop. Sci. 2011, 16, 424-432. [CrossRef] [PubMed]

7. Dellaroza, M.S.G.; Pimenta, C.A.M.; Matsuo, T. Prevalência e caracterização da dor crônica em idosos não institucionalizados. Cad. Saude Publica 2007, 23, 1151-1160. [CrossRef] [PubMed]

8. Pai, L.-W.; Hung, C.-T.; Li, S.-F.; Chen, L.-L.; Chung, Y.-C.; Liu, H.-L. Musculoskeletal pain in people with and without type 2 diabetes in Taiwan: A population-based, retrospective cohort study. BMC Musculoskelet. Disord. 2015, 16, 364. [CrossRef] [PubMed]

9. Baysal, O.; Durmus, B.; Ersoy, Y.; Altay, Z.; Senel, K.; Nas, K.; Ugur, M.; Kaya, A.; Gur, A.; Erdal, A.; et al. Relationship between psychological status and disease activity and quality of life in ankylosing spondylitis. Rheumatol. Int. 2011, 31, 795-800. [CrossRef] [PubMed]

10. Suri, P.; Morgenroth, D.C.; Kwoh, C.K.; Bean, J.F.; Kalichman, L.; Hunter, D.J. Low back pain and other musculoskeletal pain comorbidities in individuals with symptomatic osteoarthritis of the knee: Data from the osteoarthritis initiative. Arthritis Care Res. 2010, 62, 1715-1723. [CrossRef] [PubMed]

11. Miranda, V.S.; deCarvalho, V.B.; Machado, L.A.; Dias, J.M. Prevalence of chronic musculoskeletal disorders in elderly Brazilians: A systematic review of the literature. BMC Musculoskelet. Disord. 2012, 13, 82. [CrossRef] [PubMed] 
12. National Institutes of Health (Internet). NIH Guide: New Directions in Pain Research: I; National Institutes of Health: Bethesda, MD, USA, 1988.

13. Gaskin, D.J.; Richard, P. The economic costs of pain in the United States. J. Pain 2012, 13, 715-724. [CrossRef] [PubMed]

14. Phillips, C.J. The Cost and Burden of Chronic Pain. Rev. Pain 2009, 3, 2-5. [CrossRef] [PubMed]

15. Tüzün, E.H. Quality of life in chronic musculoskeletal pain. Best Pract. Res. Clin. Rheumatol. 2007, 21, 567-579. [CrossRef] [PubMed]

16. Bingefors, K.; Isacson, D. Epidemiology, co-morbidity, and impact on health-related quality of life of self-reported headache and musculoskeletal pain-a gender perspective. Eur. J. Pain 2004, 8, 435-450. [CrossRef] [PubMed]

17. Moulin, D.E. Systemic drug treatment for chronic musculoskeletal pain. Clin. J. Pain 2001, 17, S86-S93. [CrossRef] [PubMed]

18. Ndlovu, M.; Bedson, J.; Jones, P.W.; Jordan, K.P. Pain medication management of musculoskeletal conditions at first presentation in primary care: Analysis of routinely collected medical record data. BMC Musculoskelet. Disord. 2014, 15, 418. [CrossRef] [PubMed]

19. Martel, M.O.; Finan, P.H.; Dolman, A.J.; Subramanian, S.; Edwards, R.R.; Wasan, A.D.; Jamison, R.N. Self-reports of medication side effects and pain-related activity interference in patients with chronic pain: A longitudinal cohort study. Pain 2015, 156, 1092-1100. [CrossRef] [PubMed]

20. Feine, J.S.; Lund, J.P. An assessment of the efficacy of physical therapy and physical modalities for the control of chronic musculoskeletal pain. Pain 1997, 71, 5-23. [CrossRef]

21. Carlson, H.; Carlson, N. An overview of the management of persistent musculoskeletal pain. Ther. Adv. Musculoskelet. Dis. 2011, 3, 91-99. [CrossRef] [PubMed]

22. Hoving, J.L.; Koes, B.W.; De Vet, H.C.W.; Van der Windt, D.A.W.M.; Assendelft, W.J.J.; Van Mameren, H.; Devillé, W.L.J.M.; Pool, J.J.M.; Scholten, R.J.P.M.; Bouter, L.M. Manual therapy, physical therapy, or continued care by a general practitioner for patients with neck pain: A randomized, controlled trial. Ann. Intern. Med. 2002, 136, 713-722. [CrossRef] [PubMed]

23. Korthals-de Bos, I.B.C.; Hoving, J.L.; van Tulder, M.W.; Rutten-van Mölken, M.P.M.; Adèr, H.J.; de Vet, H.C.W.; Koes, B.W.; Vondeling, H.; Bouter, L.M. Cost effectiveness of physiotherapy, manual therapy, and general practitioner care for neck pain: Economic evaluation alongside a randomised controlled trial. BMJ 2003, 326, 911. [CrossRef] [PubMed]

24. Zou, L.; Wang, H.; Xiao, Z.; Fang, Q.; Zhang, M.; Li, T.; Du, G.; Liu, Y. Tai chi for health benefits in patients with multiple sclerosis: A systematic review. PLoS ONE 2017, 12, e0170212. [CrossRef] [PubMed]

25. Zou, L.; Sasaki, J.E.; Wang, H.; Xiao, Z.; Fang, Q.; Zhang, M. A systematic review and meta-analysis of Baduanjin Qigong for health benefits: Randomized controlled trials. Evid. Based Complement. Altern. Med. 2017, 2017, 1-17. [CrossRef] [PubMed]

26. Zou, L.; Wang, C.; Tian, Z.; Wang, H.; Shu, Y. Effect of Yang-Style Tai Chi on Gait Parameters and musculoskeletal flexibility in healthy Chinese older women. Sports 2017, 5, 52. [CrossRef]

27. Zou, L.; Wang, C.; Chen, K.; Shu, Y.; Chen, X.; Luo, L.; Zhao, X. The effect of Taichi practice on attenuating bone mineral density loss: A systematic review and meta-analysis of randomized controlled Trials. Int. J. Environ. Res. Public Health 2017, 14, 1000. [CrossRef] [PubMed]

28. Zou, L.; Wang, C.; Wang, H. Effect of a long-term modified Tai Chi-based intervention in attenuating bone mineral density in postmenopausal women in southeast China: Study protocol for a randomized controlled trial. Clin. Trials Degener Dis. 2017, 2, 46-52. [CrossRef]

29. Zou, L.; Xiao, Z.; Wang, H. Martial arts for health benefits in children and youth with autism spectrum disorder: A systematic review. Arch. Budo 2017, 13, 79-92.

30. Lou, L.; Zou, L.; Fang, Q.; Wang, H.; Liu, Y.; Tian, Z.; Han, Y. Effect of Taichi softball on function-related outcomes in older adults: A randomized control trial. Evid. Based Complement. Altern. Med. 2017, 2017. [CrossRef] [PubMed]

31. Zou, L.; Pan, Z.; Yeung, A.; Talwar, S.; Wang, C.; Liu, Y.; Shu, Y.; Chen, X.; Thomas, G.A. A review study on the beneficial effects of Baduanjin. J. Altern. Complement. Med. 2017. [CrossRef] [PubMed]

32. Wang, C.; Schmid, C.H.; Iversen, M.D.; Harvey, W.F.; Fielding, R.A.; Driban, J.B.; Price, L.L.; Wong, J.B.; Reid, K.F.; Rones, R.; et al. Comparative effectiveness of Tai Chi versus physical therapy for knee osteoarthritis: A randomized trial. Ann. Intern. Med. 2016, 165, 77-86. [CrossRef] [PubMed] 
33. Zou, L.; Wang, C. Traditional Chinese Baduanjin Qigong for older adults: A mini-review. Open Access J. Gerontol. Geriatr. Med. 2017, 1, 555561.

34. Zou, L.; Cui, Y.; Wang, H.; Li, T.; Lu, M.; Wang, C. Effects of traditional Chinese mind-body exercise on disease activity, spinal mobility, and quality of life in patients with ankylosing spondylitis. Trav. Hum. 2017, 80, 1587-1597.

35. Antonishen, K. Exercise mode heterogeneity among reported studies of the qigong practice Baduanjin. J. Bodyw. Mov. Ther. 2015, 19, 278-283. [CrossRef] [PubMed]

36. Hsu, M.-C.; Wang, T.-S.; Liu, Y.-P.; Liu, C.-F. Effects of Baduanjin exercise on oxidative stress and antioxidant status and improving quality of life among middle-aged women. Am. J. Chin. Med. 2008, 36, 815-826. [CrossRef] [PubMed]

37. Du, H.; Liu, J.; Qin, J. Effect of Baduanjin on pain relief in people with discogenic low back pain. Chin. J. Conval. Med. 2017, 26, 482-484.

38. Wang, C.; Guo, Z.; Chen, Z. Study on the effects of Baduanjin exercise on patients with scapulohumeral pariarthritis. Chin. J. Sports Med. 2010, 29, 285-287.

39. Cai, J.; Xiao, H.; Shi, X.; Liu, T. Effect of Baduanjin on pain in patients with nerve-root type cervical spondylosis. Guid. J. Tradit. Chin. Med. Pharm. 2015, 21, 98-99.

40. Li, C.; He, S.; Wu, B.; Wang, L. The effects of Baduanjin exercise on pain and flexibility in people with scapulohumeral periarthritis. Liaoning Sports Sci. Technol. 2010, 32, 43-52.

41. Xu, H.; Chen, X.; Ji, C.; Zhang, W.; Huang, X.; Lu, Y. Effects of Baduanjin exercise on serum interleukin-1 beta, interleukin-6, and tumor necrosis factor-alpha in patients with lumbar disc herniation. Hunan J. Tradit. Chin. Med. 2015, 31, 16-18.

42. Chen, Y.; Xiong, X.; Liu, H.; Liu, X.; Chen, Y.; Wang, S.; Xiong, Q.; Wen, Y. Effect of Baduanjin on musculoskeletal pain and quality of life in older adults. China Naturop. 2017, 25, 18-19.

43. Da Costa, B.R.; Hilfiker, R.; Egger, M. PEDro's bias: Summary quality scores should not be used in meta-analysis. J. Clin. Epidemiol. 2013, 66, 75-77. [CrossRef] [PubMed]

44. Wer, R.; Li, Y.; Guo, Y.; Yin, Z. Effects of Baduanjin on physical function and pan in people with ankylosing spondylitis. J. North Med. 2012, 9, 67-68.

45. Wan, J.; Liang, Z.; Sun, K.; Wu, J.; Ding, X. Combination of Gongfa exercise Baduanjin and manipulation for the treatment of myofascial pain syndrome on shoulder and neck. Shanghai J. Tradit. Chin. Med. 2013, $47,54-56$.

46. Wang, C.; Guo, Z.; Chen, Z. Effect of Baduanjin on disease-specific symptoms in patients with scapulohumeral periarthritis. Chin. J. Conval. Med. 2009, 18, 1074-1076.

47. Fu, J.; Tang, R.; Li, X.; Tian, X. The influence of Baduanjin on somatic function and mental health in patients with ankylosing spondylitis. West. J. Tradit. Chin. Med. 2016, 29, 124-126.

48. Li, L.; Feng, Z. Clinical observation of treating chronic nonspecific low back pain with Baduanjin combining sling exercise therapy. Rheum. Arthritis 2015, 4, 16-19.

49. Liu, H.; Chen, Y.; Yi, X.; Zhang, Y.; Zhou, Q.; Yu, L. Effect of Baduanjin on physical and mental regulation in patients with type 2 diabetic mellitus with anxiety. Hunan J. Tradit. Chin. Med. 2014, 30, 16-18.

50. Wang, C.; Liu, L. Effect of 4-week Baduanjin on musculoskeletal pain in patients with scapulohumeral periarthritis. World J. Sports 2009, 5, 24-26.

51. Xiao, B.; Yuan, S.; Fang, L. Effect of Baduanjin on pain relief in patients with patients with cervical spondylotic radiculopathy. Clin. Res. 2003, 19, 11-12.

52. Ding, Y.; Wang, J. Effect of Baduanjin on pain in people with chronic low back pain. Chin. J. Gerontol. 2014, 29, 151-152.

53. Shang, Q. Effect of Baduanjin on pain in patients with lumbar disc herniation. China Foreign Med. Treat. 2014, 29, 151-152.

54. Xuan, S.; Tao, S.; He, T.; Luo, Y.; Wang, J.; Duan, X.; Zhou, L.; Ding, J.; Song, Y.; Chu, X. Effect of Baduanjin on symptoms in patients with chronic neck pain. J. Sichuan Tradit. Chin. Med. 2016, 34, 188-190.

55. Peng, X.; Li, Q.; Li, X. The efficacy of sitting Baduanjin exercise on lumbago and back pain of elderly patients with osteoporosis. J. Nurs. Sci. 2015, 20, 4-6.

56. An, B.; Dai, K.; Zhu, Z.; Wang, Y.; Hao, Y.; Tang, T.; Yan, H. Baduanjin alleviates the symptoms of knee osteoarthritis. J. Altern. Complement. Med. 2008, 14, 167-174. [CrossRef] [PubMed] 
57. Wang, J.Y.; Guo, H.; Tang, L.; Meng, J.; Hu, L.Y. Case-control study on regular Ba Duan Jin practice for patients with chronic neck pain. Int. J. Nurs. Sci. 2014, 1, 360-366. [CrossRef]

58. Xiao, C.M.; Zhuang, Y.C. Effect of health Baduanjin Qigong for mild to moderate Parkinson's disease. Geriatr. Gerontol. Int. 2016, 16, 911-919. [CrossRef] [PubMed]

59. Chan, J.S.M.; Ho, R.T.H.; Chung, K.F.; Wang, C.W.; Yao, T.J.; Ng, S.M.; Chan, C.L.W. Qigong exercise alleviates fatigue, anxiety, and depressive symptoms, improves sleep quality, and shortens sleep latency in persons with chronic fatigue syndrome-like illness. Evid. Based Complement. Altern. Med. 2014, 2014. [CrossRef] [PubMed]

60. Wang, F.; Wang, W.; Zhang, R.; Lin, Y.; Hong, L.; Zhao, Y.; Ni, Q. Influence of different Qigong practices on sleep quality in patients with type 2 diabetes accompanied by insomnia. J. Beijing Univ. Tradit. Chin. Med. 2009, 32, 636-640.

61. Xiong, G.; Sun, H. Clinical research of Baduanjin combined with Ziwuliuzhu Nazhi acupuncture method in treatment of insomnia. China Med. Pharm. 2016, 6, 48-50.

62. Chen, Y.; Liu, R.; He, R. Effect of Baduanjin on sleep quality in older people with hypertension. Hunan J. Tradit. Chin. Med. 2015, 31, 52-54.

63. Li, L.; Wang, N. Research progress of Baduanjin in the treatment of insomnia patients with type 2 diabetes. Neimenggu Chin. Med. 2014, 27, 86-87.

64. Xiong, G.; Liu, X. Influence research of Baduanj in combined with acupuncture in insomnia (deficiency type of heart and spleen). China Med. Pharm. 2015, 5, 65-67.

65. An, B.; Wang, Y.; Jiang, X.; Lu, H.; Fang, Z.; Wang, Y.; Dai, K. Effects of Baduanjin ( 八段锦 ) exercise on knee osteoarthritis: A one-year study. Chin. J. Integr. Med. 2013, 19, 143-148. [CrossRef] [PubMed]

66. Matos, L.C.; Sousa, C.M.; Goncalves, M.; Gabriel, J.; MacHado, J.; Greten, H.J. Qigong as a traditional vegetative biofeedback therapy: Long-term conditioning of physiological mind-body effects. BioMed Res. Int. 2015, 2015. [CrossRef] [PubMed]

(C) 2018 by the authors. Licensee MDPI, Basel, Switzerland. This article is an open access article distributed under the terms and conditions of the Creative Commons Attribution (CC BY) license (http:/ / creativecommons.org/licenses/by/4.0/). 